\title{
Barriers Facing Clinical Nurse Educators' and Nursing Students' Opinion Related to
}

\section{Teaching Critical Thinking}

\section{Nawal M. Mohammed ${ }^{1}$, Samar H. Ghadery ${ }^{2}$, Heba K. Obied ${ }^{3}$}

${ }^{1}$ Demonstrator of Nursing Service Administration, , Faculty of Nursing, Tanta University

${ }^{2}$ Professor of Nursing Service Administration, Faculty of Nursing, Tanta University

${ }^{3}$ Assistant Professor of Nursing Service Administration, Faculty of Nursing, Tanta University

\section{Abstract}

Background: Critical thinking and learning are interrelated as nursing students must think to gain knowledge to be able to add to the depth and breadth of their knowledge, to become more aware of the cognitive processes and adapt personally and professionally to change' demand in nursing fields. Aim: This study aimed to assess barriers facing clinical nurse educators and nursing students' opinion related to teaching critical thinking. Subjects and Method: Research design: Descriptive cross sectional research design was used in the study. Setting: The study was conducted at Faculty of Nursing, Tanta University. The study subject: Consisted of 85 clinical nurse educators working in all departments and 464 nursing students from all academic years. Tools: Two tools were used to collect the data. Tool I: Barriers Facing Clinical Nurse Educators Related to Teaching Critical Thinking Questionnaire. Tool II: Nursing Students' Opinion about Barriers of Teaching Critical Thinking Questionnaire. Results: Majority of clinical nurse educators had low opinion level regarding total teaching critical thinking barriers. Also, nearly half of nursing students had low opinion level regarding total teaching critical thinking barriers. But, above two fifths of nursing students had moderate opinion level regarding total teaching critical thinking barriers. Conclusion: Majority of clinical nurse educators had low level clinical nurse educator, educational policy and educational curriculum related teaching critical thinking barriers. But more than two fifths of nursing students had moderate obstacles level regarding educational curriculum and faculty environment as teaching critical thinking barrier Recommendations: Providing a comfortable learning environment that facilitates for clinical nurse educators to implement critical thinking strategies such as large classes, proper furniture and provide needed equipment and supply classroom with enough internet and access. Keywords: Barriers of teaching critical thinking, Critical thinking, Nursing education, Nursing Student. 


\section{Introduction}

Critical thinking (CT) is a fundamental component in nursing as it is essential for adaptation to the everyday personal, social, and professional demands of the $21^{\text {st }}$ Century and thereafter. Rapidly changing world and the new global realities make a vital need for nursing students to develop skills to enable them to respond and adapt to these changes ${ }^{(1)}$.

The United State National Council for Excellence in Critical Thinking defines critical thinking as an intellectually disciplined process of actively and skillfully conceptualizing, applying, analyzing, synthesizing, or evaluating information gathered from, or generated by, observation, experience, reflection, reasoning, or communication, as a guide to belief and action ${ }^{(2)}$.

Although, introducing the concept of critical thinking into nursing education is a turning point in the process of professionalization, there are many barriers to implement critical thinking teaching strategies in classroom. Those barriers include student-related barriers, clinical educator-related barriers, educational curriculum-related barriers, educational policy-related barriers and university environment- related barriers $^{(38)}$.

Student -related barriers include lack of respect for some students' opinions, ambiguity of the importance of critical thinking among students and students' weak ability to distinguish between information and inappropriate conclusions $^{(3)}$.

Clinical educator-related barriers include avoid asking questions that give rise to real thinking to prevent student argument regarding sustain problems. Also, heavy burden placed on clinical educators prevents them from preparing for critical thinking, lack of their knowledge of what critical thinking is and stop reading and seeing what is new in their work field ${ }^{(4,5)}$. Educational curriculum-related barriers encompass teaching strategies that don't build on the basis of the integration of thinking skills in education. Curriculum design leads to memorization of knowledge not appropriate to develop critical thinking skills ${ }^{(6)}$.

Educational policy-related barriers occur when faculty has traditional organization of the curriculum and quota and lack of a clear educational policy in the field of introducing thinking skills in education. Moreover, standards based assessment does not lend itself to the application of student' critical thinking skills ${ }^{(5-8)}$. Faculty environmentrelated barriers include non-attractive environment for learners such as inappropriate lighting, ventilation and quiet. Furthermore, these barriers include absence of means and technologies of modern education and internet, lack of suitable 
places and lack of appropriate instructional material $^{(6)}$.

Critical thinking become a vital element in nursing education and nursing practice where nurses need to pass analysis, interpretation, inference, explanation, evaluation and self-regulation skills. These skills are required to use their holistic nursing knowledge base to think through each situation to provide individualized, effective care, not simply follow routine procedures ${ }^{(9)}$. So, it is hoped that this study will assess the barriers of teaching critical thinking from nursing students' and clinical nurse educators' opinion to overcome these barriers in the future.

\section{Aim of the study}

Assess barriers facing clinical nurse educators' and nursing students' opinion related to teaching critical thinking.

\section{Research Question:}

What are the barriers facing clinical educators and nursing students' opinion related to teaching critical thinking?

\section{Subjects and Method}

\section{Study design}

A descriptive cross sectional research design was used in present study to assess barriers facing clinical nurse educators and nursing students' opinion related to teaching critical thinking. It is a scientific method which involves assessment of barriers facing the subjects without influencing it at any way ${ }^{(10)}$.

\section{Setting}

The present study was conducted at Faculty of Nursing, Tanta University, which constructed at $1982 / 1983$ as the High Institute of Nursing, then converted officially to Faculty of Nursing at $29 / 4 / 2000$ and added to faculties of nursing in Egypt.

The Faculty consisted of six academic nursing departments, namely medicalsurgical nursing, obstetric \& gynecological nursing, pediatric nursing, community health nursing, nursing services administration and psychiatric \& mental health nursing. The capacity of the faculty was 1301 nursing students in 2019; the numbers were for first year 232, second year 292, third year 360, and fourth year $417^{(11)}$. The total number of clinical educators during time of data collection was $(94)^{(12)}$.

\section{Subjects}

All clinical nurse educators working in all departments at Faculty of Nursing -Tanta University (No.=85) who are responsible for students training in clinical areas as(52) nursing demonstrators and (42) assistant lectures.

Representative sample of nursing students (35\%) at 95\% confidence level and 90\% power of the study from each academic 
year included in the study from first year $(n=81)$, second year $(n=102)$, third year $(n=126)$, and fourth year $(n=155)$. Total sample was 464 .

\section{Tools}

To achieve the aim of present study, the data was collected using the following tools:

Tool I: Barriers Facing Clinical Nurse Educators Related to Teaching Critical Thinking Questionnaire:

This tool was modified by the researcher to collect the data about clinical nurse educators' barriers of teaching critical thinking to nursing students depend on Aliakbari and Sadeghda (2010) (13), Dickson Grosser (2012) ${ }^{(14)}$.

It consisted of two parts as follow:

Part one: Clinical nurse educator characteristic data included age, sex, years of experience, qualification, department, number of students per clinical session.

Part two: Barriers facing clinical nurse educators related to teaching critical thinking questionnaire. It consisted of 62 items classified into five dimensions;

1-Student - related barriers included (18 items).

2- Clinical educator- related barriers included (20 items).

3- Educational policy- related barriers included (10 items).

4- Educational curriculum- related barriers included (11 items).
5- Faculty environment- related barriers included ( 3 items).

\section{Scoring system}

Clinical nurse educators' responses for this part was measured on a three points Likert Scale ranging from (3) agree to (1) disagree. The total scores was calculated by summing of all categories where:

- High barriers level of teaching critical thinking $\geq 75 \%$

- Moderate barriers level of teaching critical thinking 60-75\%

- Low barriers level of teaching critical thinking $\leq 60 \%$

\section{Tool II: Nursing Students' Opinion} about Barriers of Teaching Critical Thinking Questionnaire:

This tool was developed by the researcher to collect the data about nursing students opinion about barriers of teaching critical thinking to nursing students guided by Aliakbari and Sadeghda (2010) (13), Dickson Grosser (2012) ${ }^{(14)}$. It consisted of two parts as follow:

Part one: Nursing students' characteristic data included age, sex, academic year, previous year grade, nursing specialty currently enrolled in, residence, attending program or activity about critical thinking.

Part two: Nursing students' opinion about barriers of teaching critical thinking in classroom questionnaire. It consisted of 45 items classified into four dimensions: 
1- Student - related critical thinking barriers included (18 items).

2- Clinical educator - related critical thinking barriers included (12 items).

3- Educational curriculum- related critical thinking barriers included ( 7 items).

4- Faculty environment- related critical thinking barriers included (3 items).

\section{Scoring system}

Nursing students' responses for this part was measured on a three points Likert Scale ranging from (3) agree to (1) disagree. The total scores was calculated by summing of all categories where:

- High level of perceived critical thinking $\geq 75 \%$.

- Moderate level of perceived critical thinking 60-75\%.

- Low level of perceived critical thinking $\leq 60 \%$.

\section{Method}

1. Official permission was obtained from Dean of Faculty of Nursing, Tanta University to conduct the study.

2. Ethical consideration: subjects' consent for participation was obtained after explanation of the nature and the purpose of the study. Confidentiality of information's obtained from them and the right to withdrawal was reserved.
3. The study tools were developed and designed by researcher based on review of the related literature.

4. The tools were reviewed with the supervisors and then tools were presented to a jury of 5 experts to check content validity of its items. The experts were five;

-Four assistant professors of Nursing Administration from Faculty of Nursing, Tanta University

- One assistant professor of Nursing Administration from Faculty of Nursing, Elmonofia University.

5. The experts' responses were represented in four points rating scale ranged from (4-1); 4= strongly relevant, $3=$ relevant, $2=$ little relevant, and $1=$ not relevant. Necessary modifications were done including; clarification, omission of certain items and adding others and simplifying work related words.

-The face validity value of tool (I) Barriers facing clinical nurse educators' related to teaching critical thinking questionnaire was $\mathbf{9 7 . 8 \%}$. Tool (2) Nursing students' opinion about teaching critical thinking in classroom questionnaire was $96 \%$.

6. A pilot study was carried out on a 
sample (10\%) of 9 clinical nurse educators and 46 nursing students, and they excluded from the main study sample during the actual collection of data. A pilot study was carried out after the experts' opinion and before starting the actual data collection. The pilot study was done to test clarity, sequence of items, applicability, relevance of the questions, and to determine the needed time to complete the questionnaire. According to feedback from pilot study, the tool was modified by the researcher. The estimated time needed to complete the questionnaire items from clinical nurse educators was 15-20 minutes, while from nursing students 10-15 minutes.

7. Reliability of tools was tested using Cronbach Alpha Coefficient test.

Reliability of tool (I) Barriers facing clinical nurse educators' related to teaching critical thinking questionnaire 0.888 , and reliability of tool (II) Nursing students' opinion about teaching critical thinking in classroom questionnaire was $=0.865$.

8. Barriers facing nursing clinical educators' related to teaching critical thinking questionnaire and Nursing students' opinion about teaching critical thinking in classroom questionnaire were used to collect data from identified subjects.

9. Data collection phase: the data were collected from clinical nurse educators and nursing students by the researcher. The researcher met the respondents' in groups consisted of ten per session during their work to distribute the questionnaires. The subjects recorded the answer in the presence of the researcher to ascertain that all questions were answered. The appropriate time for data collection was according the type of work and work load for each department; sometimes, it was in the morning before clinical day and other time after clinical day. The data was collected over period of three months started from February until April 2019.

\section{Statistical analysis}

The collected data were organized, tabulated and statistically analyzed using SPSS version 19 (Statistical Package for Social Studies) created by IBM, Illinois, Chicago, USA. For categorical variable the number and percentage were calculated and differences between subcategories were tested by chi square $\left(X^{2}\right)$. When chi square was not appropriate, Monte Carlo exact test. The correlation between two variables was 
calculated using Pearson's correlation coefficient. The level of significant was adopted at $\mathrm{p}<0.05$.

\section{Results}

Table (1): shows personal characteristics of clinical nurse educators. Highest percent $(45.9 \%)$ of clinical nurse educators fall in age group $>25-30$ years with mean age $28.93 \pm 3.17$. Nearly all $(98.8 \%)$ of them were female.

With regard position, around half $(50.6 \%)$ of clinical nurse educators were demonstrators and more than half $(54.1 \%)$ of them had between 5 to less than 10 years of experience, with mean score 5.14 \pm 2.78 years of experience. Around quarter $(23.5 \%)$ of clinical nurse educators were from medical and surgical nursing department. The clinical nurse educators in clinical sessions work with groups of nursing students ranged between 11.075.0 students, with mean scores $24.49 \pm$ 11.34 and more than one quarter $(29.4 \%)$ of them worked with students in groups more than or equal 30 students.

Table (2): Shows personal characteristics of nursing students. Highest percent (43.5\%) of them fall in age group $\geq 22$ years. Nursing students' age ranged between 19-23years with mean scores $21.02 \pm 1.25$. More than half $(54.5 \%)$ of nursing students were female and one third $(33.4 \%)$ of them were from fourth academic year.
More than one third (39.4\%) of nursing students enrolled in medical and surgical nursing specialty, and the highest percent) $38.8 \%$ ) of them had very good previous year grade. More than half $(58.2 \%)$ of nursing students were from rural area. Majority (90.3\%) of them didn't attend any program about critical thinking before.

Figure (1): Illustrates clinical nurse educators' and nursing students' opinion about total levels of teaching critical thinking barriers. This figure shows that majority $(84.7 \%)$ of clinical nurse educators and nearly half $(46.3 \%)$ of nursing students had low opinion level regarding total teaching critical thinking barriers. But, above two- fifths (43.1\%) of nursing students had moderate opinion level regarding total teaching critical thinking barriers.

Figure (2): Represents clinical nurse educators' and nursing students' opinion levels about student as teaching critical thinking barrier subscale. This figure shows that high percent $(74.1 \%)$ of nursing students had low opinion level regarding student as teaching critical thinking barrier subscale. Although, more than half $(54.1 \%)$ of clinical nurse educators had moderate opinion level regarding student as teaching critical thinking barrier subscale. 
Figure (3): Represents clinical nurse educators' and nursing students' opinion levels about clinical educator as teaching critical thinking barrier subscale. This figure shows that majority $(84.7 \%)$ of clinical educators had low opinion level regarding clinical educators as teaching critical thinking barrier subscale. Also, more than half $(56.5 \%)$ of nursing students had low opinion level and onequarter $(25.2 \%)$ of them had moderate opinion level regarding clinical educators as teaching critical thinking barrier subscale.

Table (3): Reveals relation between nursing students' opinion about of teaching critical thinking questionnaire and barriers facing clinical nurse educators related to teaching critical thinking. This table shows that there is a positive statistical significant relation between nursing students' opinion about of teaching critical thinking and barriers facing clinical nurse educators related to teaching critical thinking questionnaire at $(\mathrm{p}=0.001)$.

Table (4): Reveals relation between nursing students' opinion about of teaching critical thinking barriers and nursing students' personal characteristics data. This table shows that there is a positive statistical significant relation between nursing students' opinion about of teaching critical thinking barriers and nursing students' personal characteristics data. There is a positive statistical significant relation between all identified component of personal characteristics data except nursing students sex and residence at $(\mathrm{p}=0.001)$.

Around two- thirds $(65.3 \%)$ of nursing students with age group $\geq 22$ years old had high opinion level regarding teaching critical thinking barriers. Around half $(53.1 \%, 46.9 \%)$ of nursing students were from $4^{\text {th }}$ academic year and with a very good previous grade, respectively had high opinion level regarding teaching critical thinking barriers. Nearly thirty percent $(28.6 \%)$ of nursing students were from administration department had high opinion level regarding teaching critical thinking barriers. Also, majority (95.9\%) of them who not attending program or activity about critical thinking had high opinion level regarding teaching critical thinking barriers. 
Table (1): Clinical nurse educators distribution regarding personal characteristics $(n=85)$.

\begin{tabular}{|c|c|c|}
\hline Personal characteristics & $\mathbf{N}$ & $\%$ \\
\hline $\begin{array}{l}\text { Age } \\
20-25 \\
\quad>25-30 \\
>30\end{array}$ & $\begin{array}{l}17 \\
39 \\
29\end{array}$ & $\begin{array}{l}20.0 \\
45.9 \\
34.1\end{array}$ \\
\hline $\begin{array}{l}\text { Range } \\
\text { Mean } \pm \mathrm{SD}\end{array}$ & \multicolumn{2}{|c|}{$\begin{array}{c}24.0-35.0 \\
28.93 \pm 3.17\end{array}$} \\
\hline $\begin{array}{l}\text { Sex } \\
\text { Male } \\
\text { Female }\end{array}$ & $\begin{array}{c}1 \\
84\end{array}$ & $\begin{array}{c}1.2 \\
98.8\end{array}$ \\
\hline $\begin{array}{l}\text { Position } \\
\text { Demonstrator } \\
\text { Assist Lecturer }\end{array}$ & $\begin{array}{l}43 \\
42\end{array}$ & $\begin{array}{l}\mathbf{5 0 . 6} \\
49.4\end{array}$ \\
\hline $\begin{array}{l}\text { Years of experience } \\
<5 \\
5-<10 \\
\geq 10\end{array}$ & $\begin{array}{c}34 \\
46 \\
5\end{array}$ & $\begin{array}{r}40.0 \\
\mathbf{5 4 . 1} \\
5.9\end{array}$ \\
\hline $\begin{array}{l}\text { Range. } \\
\text { Mean } \pm \text { SD }\end{array}$ & \multicolumn{2}{|c|}{$\begin{array}{c}1.0-13.0 \\
5.14 \pm 2.78\end{array}$} \\
\hline $\begin{array}{l}\text { Academic nursing department } \\
\text { Administration } \\
\text { Pediatrics } \\
\text { Medical and surgical } \\
\text { Psychiatry } \\
\text { Community } \\
\text { Gynecology and obstetric }\end{array}$ & $\begin{array}{c}16 \\
13 \\
20 \\
8 \\
12 \\
16\end{array}$ & $\begin{array}{c}18.8 \\
15.3 \\
\mathbf{2 3 . 5} \\
9.4 \\
14.2 \\
18.8\end{array}$ \\
\hline $\begin{array}{l}\text { Number of student per clinical session } \\
10-<20 \\
20-<30 \\
\geq 30\end{array}$ & $\begin{array}{l}33 \\
27 \\
25\end{array}$ & $\begin{array}{l}38.8 \\
31.8 \\
29.4\end{array}$ \\
\hline $\begin{array}{l}\text { Range } \\
\text { Mean } \pm \mathrm{SD}\end{array}$ & \multicolumn{2}{|c|}{$\begin{array}{c}11.0-75.0 \\
24.49 \pm 11.34\end{array}$} \\
\hline
\end{tabular}


Table (2): Nursing students distribution regarding personal characteristics of $(n=464)$

\begin{tabular}{|c|c|c|}
\hline Personal characteristics & $\mathbf{N}$ & $\%$ \\
\hline \multicolumn{3}{|l|}{ Age } \\
\hline$<20$ & 81 & 17.5 \\
\hline $20-<22$ & 181 & 39.0 \\
\hline$\geq 22$ & 202 & 43.5 \\
\hline Min. - Max. & \multirow{2}{*}{\multicolumn{2}{|c|}{$\begin{array}{c}19.0-23.0 \\
21.02 \pm 1.25\end{array}$}} \\
\hline Mean \pm SD & & \\
\hline \multicolumn{3}{|l|}{$\operatorname{Sex}$} \\
\hline Male & 211 & 45.5 \\
\hline Female & 253 & 54.5 \\
\hline \multicolumn{3}{|l|}{ Academic year } \\
\hline $1^{\text {st }}$ & 81 & 17.5 \\
\hline $2^{\text {nd }}$ & 102 & 22.0 \\
\hline $3^{\text {rd }}$ & 126 & 27.1 \\
\hline $4^{\text {th }}$ & 155 & 33.4 \\
\hline \multicolumn{3}{|c|}{ Nursing specialty currently enrolled in } \\
\hline Administration & 77 & 16.7 \\
\hline Pediatrics & 72 & 15.5 \\
\hline Medical And Surgical & 183 & 39.4 \\
\hline Communications & 78 & 16.8 \\
\hline Gynecology and obstetric & 54 & 11.6 \\
\hline \multicolumn{3}{|l|}{ Previous year grade } \\
\hline No (1st year) & 81 & 17.5 \\
\hline Excellent & 128 & 27.6 \\
\hline Very good & 180 & 38.8 \\
\hline Good & 67 & 14.4 \\
\hline Fair & 8 & 1.7 \\
\hline \multicolumn{3}{|l|}{ Residence } \\
\hline Urban & 194 & 41.8 \\
\hline Rural & 270 & 58.2 \\
\hline \multicolumn{3}{|c|}{ Attending program or activity about critical thinking } \\
\hline Yes & 45 & 9.7 \\
\hline No & 419 & 90.3 \\
\hline
\end{tabular}




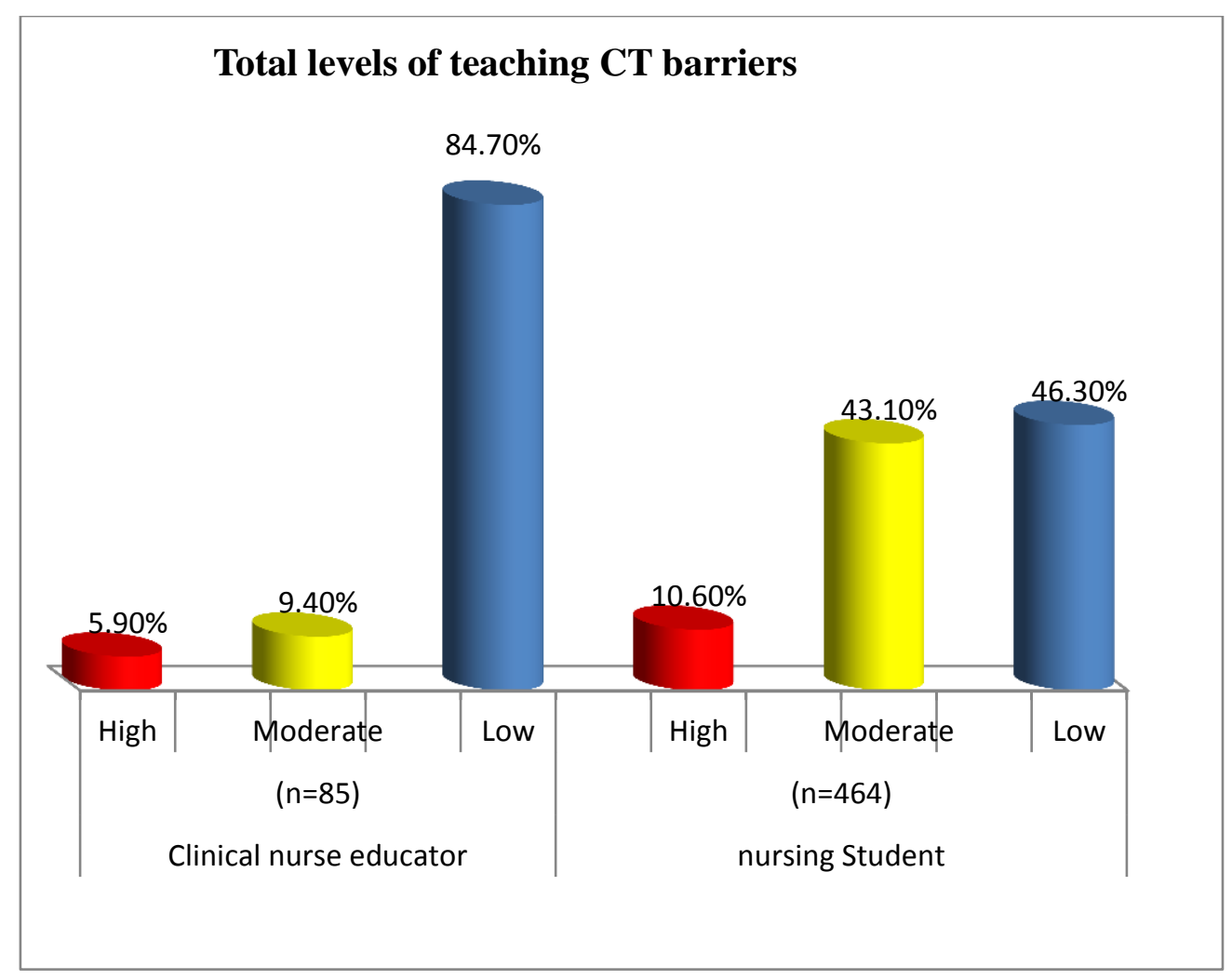

Figure (1): Clinical nurse educators' and nursing students' opinion about total levels of teaching critical thinking barriers. 


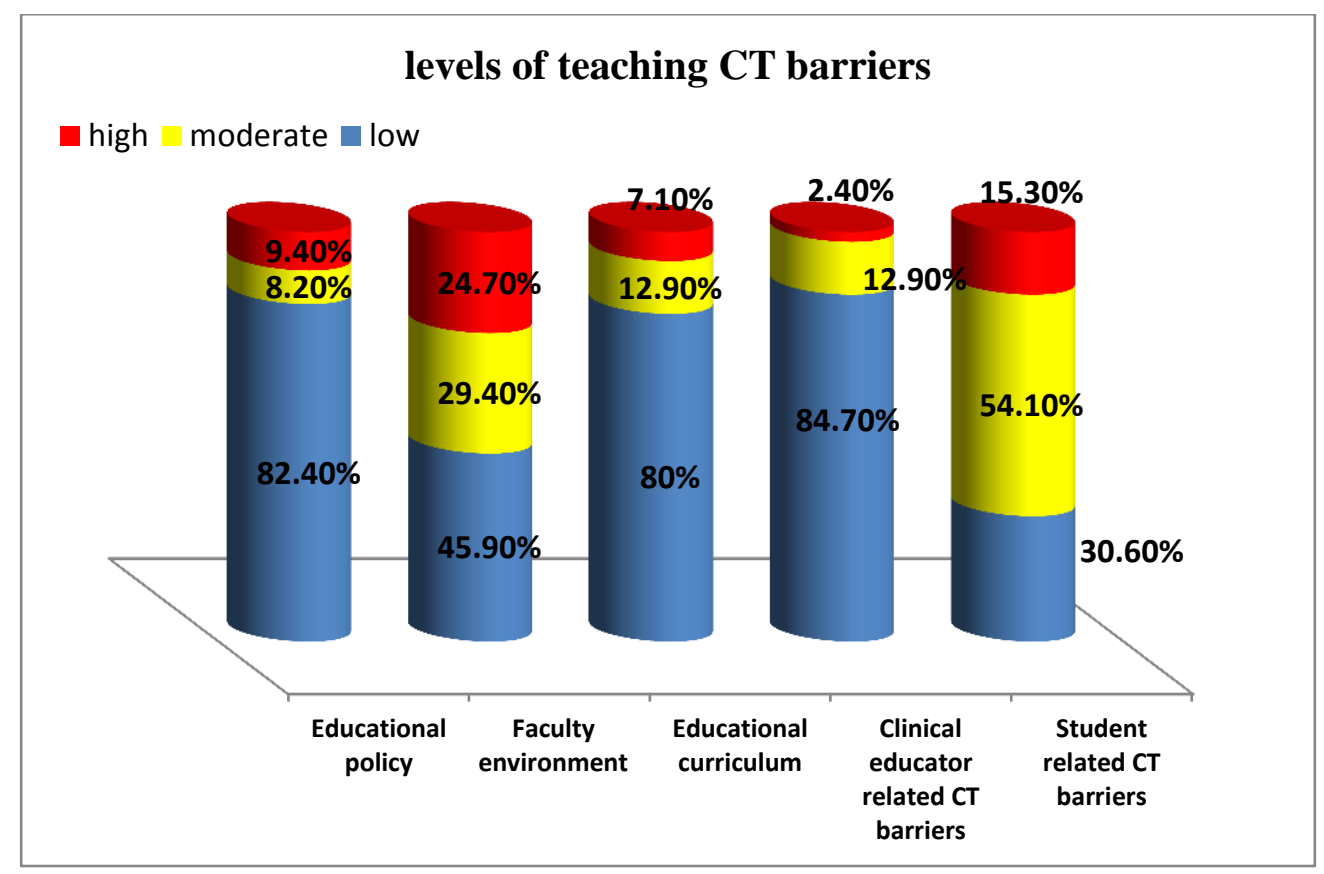

Figure (2): Levels of teaching critical thinking barriers facing clinical nurse educators $(\mathbf{n}=\mathbf{8 5})$

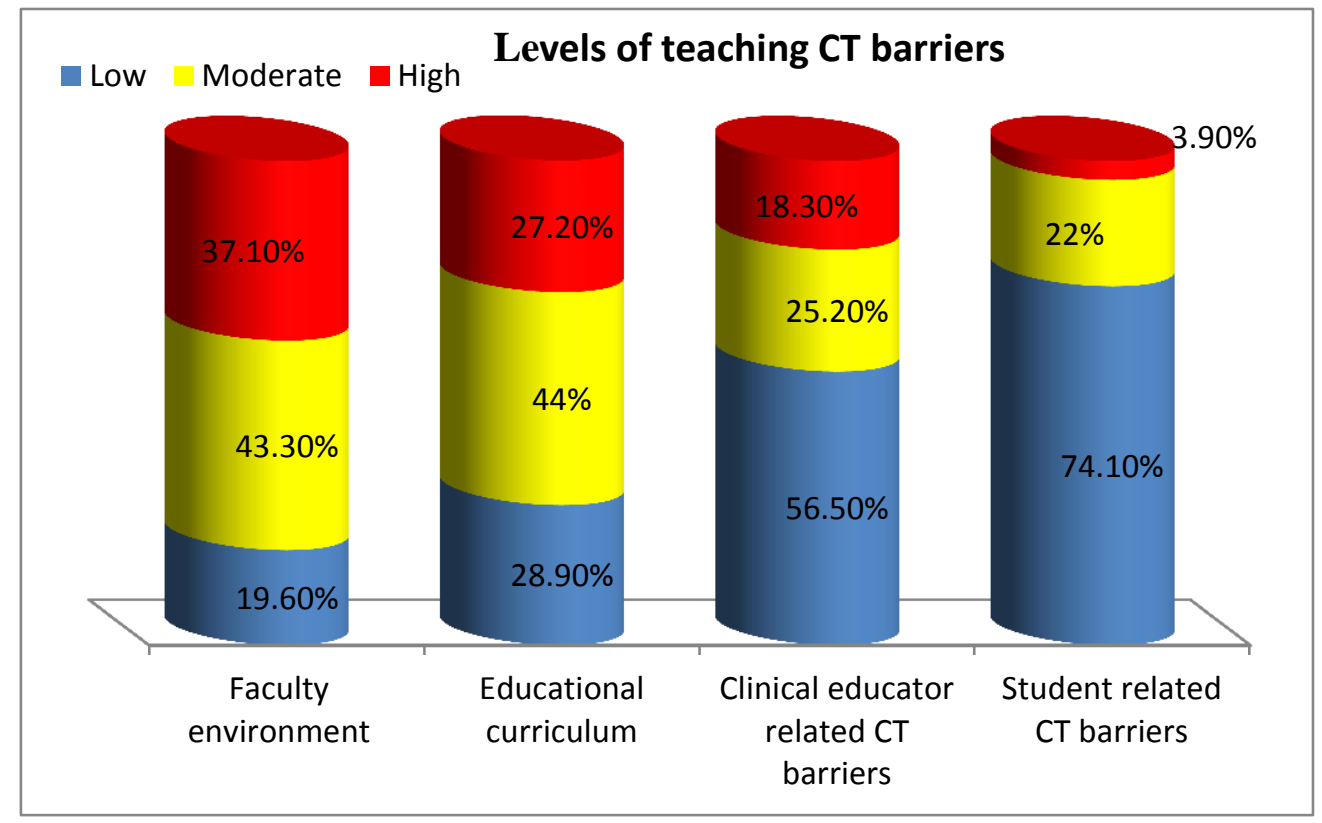

Figure (3): Levels of nursing students' opinion about teaching critical thinking barriers. 
Table (3): Correlation between nursing students' opinion about teaching critical thinking and barriers facing clinical nurse educators related to teaching critical thinking

\begin{tabular}{|c|c|c|c|c|c|c|}
\hline \multirow{2}{*}{\multicolumn{2}{|c|}{$\begin{array}{l}\text { Nursing students' } \\
\text { Barriers facing } \\
\text { clinical nurse educators }\end{array}$}} & \multicolumn{5}{|c|}{$\begin{array}{l}\text { Nursing students' opinion about teaching critical thinking } \\
\text { barriers }\end{array}$} \\
\hline & & $\begin{array}{l}\text { Student } \\
\text { related } \\
\text { teaching } \\
\text { CT } \\
\text { barriers }\end{array}$ & $\begin{array}{c}\text { Clinical } \\
\text { educator } \\
\text { related } \\
\text { teaching CT } \\
\text { barriers }\end{array}$ & \begin{tabular}{|} 
Educational \\
curriculum \\
related \\
teaching CT \\
barriers
\end{tabular} & $\begin{array}{c}\text { Faculty } \\
\text { environment } \\
\text { related } \\
\text { teaching CT } \\
\text { barriers }\end{array}$ & Total \\
\hline $\begin{array}{l}\text { Student related teaching } \\
\text { CT barriers }\end{array}$ & $\mathbf{r}$ & $\begin{array}{l}0.148^{*} \\
0.001^{*}\end{array}$ & $\begin{array}{l}0.201^{*} \\
<0.001^{*}\end{array}$ & $\begin{array}{l}0.153^{*} \\
0.001^{*}\end{array}$ & $\begin{array}{l}0.237^{*} \\
<0.001^{*}\end{array}$ & $\begin{array}{l}0.173^{*} \\
<0.001^{*}\end{array}$ \\
\hline $\begin{array}{l}\text { Clinical educator } \\
\text { related teaching CT } \\
\text { barriers }\end{array}$ & $\mathbf{p}$ & $\begin{array}{l}0.119^{*} \\
0.010^{*}\end{array}$ & $\begin{array}{l}0.163^{*} \\
<0.001^{*}\end{array}$ & $\begin{array}{l}0.119^{*} \\
0.010^{*}\end{array}$ & $\begin{array}{l}0.174^{*} \\
<0.001^{*}\end{array}$ & $\begin{array}{l}0.138^{*} \\
0.003^{*}\end{array}$ \\
\hline $\begin{array}{l}\text { Educational curriculum } \\
\text { related teaching CT } \\
\text { barriers }\end{array}$ & p & $\begin{array}{l}0.009 \\
0.851\end{array}$ & $\begin{array}{l}0.025 \\
0.588\end{array}$ & $\begin{array}{l}-0.003 \\
0.947\end{array}$ & $\begin{array}{l}0.039 \\
0.400\end{array}$ & $\begin{array}{l}0.014 \\
0.767\end{array}$ \\
\hline $\begin{array}{l}\text { Faculty environment } \\
\text { related teaching CT } \\
\text { barriers }\end{array}$ & $\mathbf{p}$ & $\begin{array}{l}0.100^{*} \\
0.031^{*}\end{array}$ & $\begin{array}{l}0.131^{*} \\
0.005^{*}\end{array}$ & $\begin{array}{l}0.098^{*} \\
0.035^{*}\end{array}$ & $\begin{array}{l}0.140^{*} \\
0.002^{*}\end{array}$ & $\begin{array}{l}0.114^{*} \\
0.014^{*}\end{array}$ \\
\hline Total & $\mathbf{r}$ & $\begin{array}{l}0.139^{*} \\
0.003^{*}\end{array}$ & $\begin{array}{l}0.193^{*} \\
<0.001^{*}\end{array}$ & $\begin{array}{l}0.137^{*} \\
0.003^{*}\end{array}$ & $\begin{array}{l}0.221^{*} \\
<0.001^{*}\end{array}$ & $\begin{array}{l}0.163^{*} \\
<0.001^{*}\end{array}$ \\
\hline
\end{tabular}

r: Pearson coefficient *: Statistically significant at $\mathbf{p} \leq \mathbf{0 . 0 5}$ 
Table (4): Relation between nursing students' opinion about teaching critical thinking barriers and personal characteristics $(n=85)$

\begin{tabular}{|c|c|c|c|c|c|c|c|c|}
\hline \multirow{3}{*}{ Personal characteristics } & \multicolumn{6}{|c|}{ Nursing students' opinion about } & \multirow{3}{*}{$x^{2}$} & \multirow{3}{*}{$\mathbf{P}$} \\
\hline & \multicolumn{2}{|c|}{ Low } & \multicolumn{2}{|c|}{ Moderate } & \multicolumn{2}{|c|}{ High } & & \\
\hline & No. & $\%$ & No. & $\%$ & No. & $\%$ & & \\
\hline \multicolumn{9}{|l|}{ Age } \\
\hline$<20$ & 34 & 15.8 & 39 & 19.5 & 8 & 16.3 & \multirow{3}{*}{18.732} & \multirow{3}{*}{$0.001^{*}$} \\
\hline $20-<22$ & 102 & 47.4 & 70 & 35.0 & 9 & 18.4 & & \\
\hline$\geq 22$ & 79 & 36.7 & 91 & 45.5 & 32 & 65.3 & & \\
\hline \multicolumn{9}{|l|}{ Gender } \\
\hline Male & 101 & 47.0 & 88 & 44.0 & 22 & 44.9 & \multirow{2}{*}{0.378} & \multirow{2}{*}{0.828} \\
\hline Female & 114 & 53.0 & 112 & 56.0 & 27 & 55.1 & & \\
\hline \multicolumn{7}{|l|}{ Academic year } & \multirow{5}{*}{45.352} & \multirow{5}{*}{$<0.001^{*}$} \\
\hline $1^{\text {st }}$ & 34 & 15.8 & 39 & 19.5 & 8 & 16.3 & & \\
\hline $2^{\text {nd }}$ & 74 & 34.4 & 28 & 14.0 & 0 & 0.0 & & \\
\hline $3^{\mathrm{rd}}$ & 45 & 20.9 & 66 & 33.0 & 15 & 30.6 & & \\
\hline $4^{\text {th }}$ & 62 & 28.8 & 67 & 33.5 & 26 & 53.1 & & \\
\hline \multicolumn{7}{|l|}{ Nursing specialty } & \multirow{6}{*}{29.709} & \multirow{6}{*}{$<0.001^{*}$} \\
\hline Administration & 31 & 14.4 & 32 & 16.0 & 14 & 28.6 & & \\
\hline Pediatrics & 29 & 13.5 & 36 & 18.0 & 7 & 14.3 & & \\
\hline Medical & 108 & 50.2 & 67 & 33.5 & 8 & 16.3 & & \\
\hline Communications & 31 & 14.4 & 35 & 17.5 & 12 & 24.5 & & \\
\hline Obstetric & 16 & 7.4 & 30 & 15.0 & 8 & 16.3 & & \\
\hline \multicolumn{7}{|l|}{ Previous grade } & \multirow{6}{*}{16.931} & \multirow{6}{*}{$0.026^{*}$} \\
\hline No $\left(1^{\text {st }}\right.$ year $)$ & 34 & 15.8 & 39 & 19.5 & 8 & 16.3 & & \\
\hline Excellent & 72 & 33.5 & 47 & 23.5 & 9 & 18.4 & & \\
\hline Very good & 75 & 34.9 & 82 & 41.0 & 23 & 46.9 & & \\
\hline Good & 32 & 14.9 & 30 & 15.0 & 5 & 10.2 & & \\
\hline Fair & 2 & 0.9 & 2 & 1.0 & 4 & 8.2 & & \\
\hline \multicolumn{7}{|l|}{ Residence } & \multirow{3}{*}{2.178} & \multirow{3}{*}{0.337} \\
\hline Urban & 83 & 38.6 & 87 & 43.5 & 24 & 49.0 & & \\
\hline Rural & 132 & 61.4 & 113 & 56.5 & 25 & 51.0 & & \\
\hline \multicolumn{9}{|c|}{ Attending program critical about critical thinking } \\
\hline Yes & 16 & 7.4 & 27 & 13.5 & 2 & 4.1 & \multirow[b]{2}{*}{6.316} & \multirow[b]{2}{*}{$0.043^{*}$} \\
\hline No & 199 & 92.6 & 173 & 86.5 & 47 & 95.9 & & \\
\hline
\end{tabular}




\section{Discussion}

Critical thinking in nursing education is a means by which nurses can use analysis, questioning, interpretation, and reflection to resolve patient care issues. It helps students to learn tasks better and solve problems that they encountered in academic and nonacademic environments (15). Critical thinking skills is regarded as an extra edge for nursing graduates these days as they will be able to keep up with the technological innovations and have better chances at employability and demonstrate more professionalism. It has been deemed as a skill that should be gained in order to meet the today's societal expectations such as quick thinking, competent, communication, and ability to resolve conflict and reconcile diverse perspectives $^{(16)}$.

Nursing education becomes increasingly focusing on teaching strategies that enhance critical thinking, decision making and problem solving skills ${ }^{(17)}$. So, this study aimed to study barriers facing clinical nurse educators and nursing students' opinion related to teaching critical thinking. This study's discussion will be categorized under barriers facing clinical nurse educators related to teaching critical thinking, nursing students' opinion about barriers of teaching critical thinking, relation between nursing students' opinion about of teaching critical thinking and barriers facing clinical nurse educators related to teaching critical thinking and relation between nursing students' opinion about of teaching critical thinking barriers and nursing students' personal characteristics data as mentioned below.

\section{Barriers facing clinical nurse educators related to teaching critical thinking}

The present study results revealed that majority of clinical nurse educators had low level regarding total teaching critical thinking barriers. Majority of them had low level of clinical nurse educator, educational policy and educational curriculum related teaching critical thinking barriers. This result may referred to majority of clinical nurse educator had knowledge about their role as a mastermind and facilitator of critical thinking and they had adequate time management skills to cover educational curriculum. Also, they had the ability to draw students' attention to the lesson and provide nursing students with ways to search for information to improve nursing students' critical thinking skills.

This result is supported by, Lee et al. (2016) $^{(18)}$ who stated that nursing students thrived in collaborative learning environment between all team members where the needs and goals were clear and acknowledged by clinical nurse educators and nursing students. As well as he stated the importance of clinical nurse educators 
to guide nursing students through a process of developing an awareness of their ability to think critically. Therefore, to act upon it, clinical nurse educators act as facilitator and nursing students need peer assistance and collaborative efforts, contrary to the traditional teacher-centered practice of limiting classroom interaction.

On contrary, Pitt et al. (2015) ${ }^{(19)}$ indicated in his study that there were many obstacles against nurses' learning of critical thinking by clinical nurse educators such as time constraints, financial constraints, the culture of the workplace that didn't accept ability to change, access to appropriate knowledge and matching of new technologies to acquire knowledge that prevent clinical nurse educators from teaching critical thinking to nursing students.

Also, Alfaro-Le Fevre (2015) ${ }^{(20)}$ found that majority of clinical nurse educators don't have time to answer students' questions, as well as they do not want to receive any questions that they not answer it or have unclear answers for it as they don't have ability to think critically and make positive discussion and not prepared to practice critical thinking.

\section{Nursing students' opinion about barriers of teaching critical thinking}

The results of the present study showed that nearly half of nursing students had low level regarding total teaching critical thinking barriers. But, more than two fifths of nursing students had moderate level regarding educational curriculum and faculty environment as teaching critical thinking barriers. These results may be due to their opinion that they had ability to make a positive discussion, distinguish between information and inappropriate conclusions, clinical nurse educator enhance their ability to learn independently but course content is too loaded.

This result is supported by DSouza et al. (2017) (21) who indicated that nursing students stressed that large classes, time constraints, and large amount of content to provide to students preclude the teaching of critical thinking. On the other hand, Ennis (2016) ${ }^{(22)}$ indicated that the most important obstacles against teaching of critical thinking in nursing education according to nursing students' opinion are conflict, lack of access to direct knowledge and experience about critical thinking and lack of interest to make positive discussion or think critically.

Finding of the present study results showed that there was a positive statistical significant correlational between nursing students' opinion about of teaching critical thinking and barriers facing clinical nurse educators related to teaching critical thinking. This may be interpreted that clinical nurse educators provide nursing students with ways to search for 
information, educational curriculum focused only the acquisition of facts, ideas, and concepts. Also, they opinion that the main barriers facing them that the textbooks do not provide activities for improving critical thinking skills and nonattractive environment for students.

On the other hand, Ahn and Yeom (2015) ${ }^{(23)}$ showed that students lack exposure to critical thinking and clinical nurse educators' lack responsibility to promote critical thinking skills in their students are main barrier of teaching critical thinking. As they didn't provide students ways to search for more information outside textbooks.

\section{Relation between nursing students' opinion about of teaching critical thinking barriers and their personal characteristics data.}

The present study results revealed that there was a positive statistical significant relation between nursing students' opinion about of teaching critical thinking barriers and nursing students' personal characteristics data. There was a positive statistical significant correlation between nursing student' age categories and teaching critical thinking barriers. High percent of nursing students with age group above twenty two years and were from fourth academic year had high obstacles level regarding teaching critical thinking barriers. This may be interpreted by their past experience as they rarely practice critical thinking in last academic years and focus mainly on how to achieve certificate. These results were confirmed by Manan and Mehmood (2015) ${ }^{(24)}$ who found that slightly more than half of nursing students aged more than twenty one years old who not interested enough to practice critical thinking skills as they not trained on how to make scientific decisions and solve problems . Also, Coleman and Willis (2015) ${ }^{(25)}$ examination-based teaching puts clinical nurse educators under the pressure of the need for covering a pre-determined set of topics in a given number of lectures that allow nursing students to pass exams have a certificate of achievement and found that fourth year students achieve success than first year ones but didn't give importance to teach critical thinking.

Finding of the present study results showed that there was a positive statistical significant correlational between nursing student' previous year grade and teaching critical thinking barriers. Around half of nursing students with a very good previous grade had high obstacles level regarding teaching critical thinking barriers. These results may be related to those nursing students prefer memorizing more than understanding and they more oriented that most of educational exams based on recalling of information rather 
understanding or thinking to achieve such high grades.

The present study results revealed that there was a positive statistical significant relation between nursing students' opinion about of teaching critical thinking barriers and previous attending of program or activity about critical thinking. Majority of them who not attending program or activity about critical thinking had high obstacles level regarding teaching critical thinking barriers. It may be related to they didn't have adequate knowledge about critical thinking and ambiguity of its importance.

The present study is supported by Frazier (2017) (26) which investigated critical thinking among undergraduate nursing students had indicated that the highest percentages of nursing students had a weak level of critical thinking skills as they had inadequate knowledge regarding critical thinking .

\section{Conclusion}

Based on the finding of the present study it was concluded that:

The majority of clinical nurse educators had low level of total teaching critical thinking barriers. Majority of them had low level of clinical nurse educator, educational policy and educational curriculum related teaching critical thinking barriers. Nearly half of nursing students had low level of total teaching critical thinking barriers. But more than two fifths of nursing students had moderate obstacles level regarding educational curriculum and faculty environment as teaching critical thinking barrier. In addition, there was a positive statistical significant correlational between nursing students' opinion about of teaching critical thinking barriers and barriers facing clinical nurse educators related to teaching critical thinking.

\section{Recommendations}

Based on the results of the present study the following recommendations were suggested for:

\section{For nursing faculty administrators:}

- Develop of nursing curriculum contents, which concentrates on improving thinking skills-in general and critical thinking skills-in specific and shouldn't be overloaded with trivial or duplicated materials.

- Transform nursing education from educator's teaching to students' learning.

- Provide adequate funding to provide training workshops, Conferences, on campus activities and materials for encouraging teaching of critical thinking in classrooms.

- Conduct in-service training programs and workshops for clinical nurse educators to enhance critical thinking skills practice education. 
- Provide orientation programs and mentors for novice nursing educators about training on classroom management with different teaching strategies for enhancing their selfcontrol.

- Conduct meetings with other faculty members and sharing their experiences for preparing group activities, assignments, exercises that can facilitate easier implementation of critical thinking.

- Provide a comfortable and enjoyable learning environment that facilitates for clinical nurse educators to implement critical thinking strategies including large classes, proper furniture and provide needed equipment and supply classroom with enough internet and access.

\section{For clinical nurse educators:}

- Create helpful classroom learning environments to encourage students actively participate in the learning process allow students interaction.

- Attending workshops and reading seminars to be proficient in implementing critical thinking strategies and other active learning activities.

- Encourage and persuade nursing students to accept and implement critical thinking in their classrooms through letting them know its benefits, group rewards.

- Relieving nursing students fear and threats regarding grading process.

- Develop more strategies for motivating students such as become a role model for nursing students, get to know students, use a variety of student active teaching activities.

\section{For nursing students:}

- Have willing and be patient about difficulty of critical thinking activities.

- Improve self-learning and ask for new ways to search information outside textbooks.

- Be aware that critical thinking skills are hallmark of effective patient care and become effective leaders.

- Maintain consistency between how to achieve high grades and improve critical thinking skills.

\section{For further studies}

- More future studies need to be encouraged for investigating efficacy of learning environment on developing critical thinking skills for the nursing students and the learning process as a general.

- Research needed to investigate relationship between teaching strategies and critical thinking skills among undergraduate nursing students in classroom as well as in their clinical 
practice.

- Critical thinking dispositions and problem solving abilities among administration nursing students.

\section{References}

1. Elfatihi M. A rationale for the integration of critical thinking skills in EFL/ESL instruction. Higher Education of Social Science. 2017; 12 (2): 26-31.

2. Jones-Schenk J, Leafman J, Wallace L, Allen P. Addressing the cost, value, and student debt in nursing education. Nurs. Econ. 2017; 35(29): 7-13.

3. Boholano H. Smart social networking: 21 st century teaching and learning skills. Research in Pedagogy. 2017; 7 (1): 21-29.

4. Kim M, Pollard V. A modest critical pedagogy for English as a foreign language education. Education as Change. 2017; 21(1): 50-72.

5. Mikkonen $\mathrm{K}$, Elo $\mathrm{S}$, Kuivila $\mathrm{H}$, Tuomikoski A, Kaariainen M. Culturally and linguistically diverse healthcare students experiences of learning in a clinical environment: A systematic review of qualitative studies. Int. J. Nurs. Studies. 2016; 54 (1): 173-187.

6. Marin M, Pava L. Conceptions of critical thinking from university EFL teachers. English Language Teaching. 2017; 10 (7): 1916-4750.
7. Ok S. Autonomy in an EFL teacher training context: Trainee teacher perceptions of instructor expectations. Australian Journal of Teacher Education. 2016; 41 (3): 66-86.

8. Papak P, Vujicic, L, ZeljkaIvkovic Z. Project activities and encouraging critical thinking: exploring teachers' attitudes. C E P S Journal. 2017; 7 (3): 27-46.

9. Shahrebabaki M, Notash M. Teachers' and learners' attitudes towards critical thinking skills: A case study in the Iranian EFL context. Journal of Applied Linguistics and Language Research. 2015; 2 (2): 93-106.

10. Oliveira L, Rueda Díaz L, Carbogim F, Rodrigues A, Püschel V. Effectiveness of teaching strategies on the development of critical thinking in undergraduate nursing students: A meta-analysis. 2016; 50(2): 350-9.

11. Nursing Students Affairs. Faculty of Nursing. Tanta University. February 2019.

12. Nursing Teaching Staff Affairs. Faculty of Nursing. Tanta University. February 2019.

13. Aliakbari L. Science production in Iranian educational system by the use of critical thinking. International Journal of Instruction. 2010; 3(1): 61-76.

14. Dickson G, Flynn L. Nurses' clinical reasoning: Processes and practices of 
medication safety. Qual. Health Res. 2012; 22(1): 3-16.

15. Swart R. Critical thinking instruction and technology enhanced learning from the student perspective: A mixed methods research study. Journal of Nurse Education Practice. 2017; 23 (1): 30-39.

16. McGrath L, Kathrin K. English for Specific Purposes and Academic Literacies: Eclecticism in academic writing pedagogy. Teaching in Higher Education. 2016; 21 (8): 933-947.

17. Ekholm E, Zumbrumm S, Conkli N. The relation of college student's selfefficacy toward writing self-regulation aptitude: Writing feedback perceptions as a mediating variable. Teaching in Higher Education. 2015; 20 (2): 197207.

18. Lee J, Lee Y, Gong S, Bae J, Choi M. A meta-analysis of the effects of nontraditional teaching methods on the critical thinking abilities of nursing students. BMC Med Educ. 2016; 16(1): 240.

19. Pitt V, Powis D, Levett-Jones T, Hunter S. The influence of critical thinking skills on performance and progression in a pre-registration nursing program. Nurse Education Today. 2015; 35(1): 125-31.

20. Alfaro R. Critical thinking and clinical judgment: A practical approach to outcome-focused thinking. 2015; 3(5):320-360.

21. Dsouza S. The importance of academic literacy for undergraduate nursing students and its relationship to future professional clinical practice: a systematic review. Nurse Educ. Today. 2017; 60(1): 84-91.

22. Ennis R. Critical thinking across the curriculum: A vision. Topoi. 2016: 1-20

23. Ahn S, Yeom H. Moral sensitivity and critical thinking disposition of nursing students in Korea. International Journal of Nursing Practice. 2015; 20(5): 482-489.

24. Manan S, Mehmood T. Culture and critical thinking in classroom: Narratives from University Students in Pakistan. Asia Pacific Journal of Social Science. 2015; 1(3):110-34.

25. Coleman D, Willis D. Reflective writing: The student nurse's perspective on reflective writing and poetry writing. Nurse Education Today. 2015; 35(7): 906-911.

26. Frazier K. Humanities, Too: In new study, history courses in critical thinking reduce pseudoscientific beliefs. Skeptical Inquirer. 2017; 41 (4): 11. 